University of New Orleans

ScholarWorks@UNO

$1-1990$

\title{
Instrument matrix of the four-detector photopolarimeter: physical meaning of its rows and columns and constraints on its elements
}

\author{
R. M.A. Azzam \\ University of New Orleans, razzam@uno.edu
}

Follow this and additional works at: https://scholarworks.uno.edu/ee_facpubs

Part of the Electrical and Electronics Commons, and the Optics Commons

\section{Recommended Citation}

R. M. A. Azzam, "Instrument matrix of the four-detector photopolarimeter: physical meaning of its rows and columns and constraints on its elements," J. Opt. Soc. Am. A 7, 87-91 (1990)

This Article is brought to you for free and open access by the Department of Electrical Engineering at ScholarWorks@UNO. It has been accepted for inclusion in Electrical Engineering Faculty Publications by an authorized administrator of ScholarWorks@UNO. For more information, please contact scholarworks@uno.edu. 


\title{
Instrument matrix of the four-detector photopolarimeter: physical meaning of its rows and columns and constraints on its elements
}

\author{
R. M. A. Azzam \\ Department of Electrical Engineering, University of New Orleans, Lakefront, New Orleans, Louisiana 70148
}

Received March 27, 1989; accepted August 29, 1989

\begin{abstract}
The four-detector photopolarimeter (FDP) is an arrangement of four photodetectors for measuring the state of polarization of light. The output current vector $\mathbf{I}$ of the FDP is related to the input Stokes vector $\mathbf{S}$ by $\mathbf{I}=\mathbf{A S}$, where $\mathbf{A}$ is the instrument matrix. The rows of $\mathbf{A}$ can be viewed as projection operators that determine the output currents of the detectors. This leads to the recognition of four special totally polarized input states, each of which maximizes the output of one detector. The associated four orthogonal states produce minimum signals. Because each detector is absorptive and its output is nonnegative, eight inequalities must be satisfied by the elements of $\mathbf{A}$. For optically isotropic detectors, one element is identically zero and another can be made zero by an appropriate coordinate rotation. Three additional inequalities that are likely to apply are also stated. The columns of $\mathbf{A}$ have the following interesting meaning. The first column represents the normalized response of the FDP for incident unpolarized light or its average response to incident light polarized in any pair of orthogonal states. The second, third, and fourth columns represent the differential normalized responses of the FDP for incident light polarized in the following pairs of orthogonal states: (1) linear polarizations at 0 and $90^{\circ}$ azimuths, (2) linear polarizations at $45^{\circ}$ and $135^{\circ}$ azimuths, and (3) the right and left circular polarizations, respectively. These are the same pairs of orthogonal states that are used in the phenomenological definitions of the Stokes parameters.
\end{abstract}

\section{INTRODUCTION}

In the four-detector photopolarimeter ${ }^{1-3}$ (FDP) the light beam, whose state of polarization is to be determined, strikes at oblique incidence three photodetector surfaces in succession, each of which is partially specularly reflecting and each of which generates an electrical signal proportional to the fraction of the radiation that it absorbs. A fourth detector captures substantially the remainder of the light. The four outputs thus developed form a $4 \times 1$ signal vector:

$$
\mathbf{I}=\left[i_{0} i_{1} i_{2} i_{3}\right]^{t}
$$

which is linearly related,

$$
\mathbf{I}=\mathbf{A S},
$$

to the input Stokes vector:

$$
\mathbf{S}=\left[S_{0} S_{1} S_{2} S_{3}\right]^{t},
$$

where $S_{0}, S_{1}, S_{2}$, and $S_{3}$ are the four Stokes parameters ${ }^{4,5}$ and $t$ indicates the transpose. Consequently, $\mathbf{S}$ is obtained by

$$
\mathbf{S}=\mathbf{A}^{-1} \mathbf{I} \text {. }
$$

The essential condition that the instrument matrix $\mathbf{A}$ be nonsingular (hence $\mathbf{A}^{-1}$ exists) requires that the plane of incidence be rotated between successive reflections by other than $90^{\circ}$, with $\pm 45^{\circ}$ and $\pm 135^{\circ}$ being the optimum rotations. ${ }^{3}$

In this paper we consider some general properties of the instrument matrix A. Physical meanings are attached to its rows and columns, and several constraints on its elements are established. The latter constraints serve as important validity checks on any theoretically or experimentally deter- mined matrix A. Calibration schemes with which $\mathbf{A}$ may be measured are discussed elsewhere. ${ }^{2,3,6}$

\section{ROWS OF A AS PROJECTION OPERATORS}

It is instructive to consider the FDP from a linear-systems viewpoint. As shown in Fig. 1, the FDP can be abstracted as a black box whose input is the Stokes vector $\mathbf{S}$ of the incident light beam and whose output is the current vector I. The instrument matrix $\mathbf{A}$, which is the essential characteristic of the black box, can be expressed in terms of its rows as

$$
\mathbf{A}=\left[\begin{array}{l}
\mathbf{A}_{0} \\
\mathbf{A}_{1} \\
\mathbf{A}_{2} \\
\mathbf{A}_{3}
\end{array}\right] .
$$

From Eqs. (1) and (2), the $m$ th output signal (current) of the $m$ th detector is

$$
i_{m}=\mathbf{A}_{m} \mathbf{S}, \quad m=0,1,2,3 .
$$

In Eq. (6), $\mathbf{A}_{m} \mathbf{S}$ is identical to the scalar product of two vectors, $\mathbf{A}_{m}{ }^{t}$ and $\mathbf{S}$, in a four-dimensional space. Therefore $i_{m}$ can be thought of as a projection of the unknown Stokes vector $\mathbf{S}$ along $\mathbf{A}_{m}{ }^{t}$. Four independent projections, which are achieved as a result of the light-beam interaction with the four detectors, are required to fix $\mathbf{S}$. Again, A must be nonsingular, and $\operatorname{det} \mathbf{A} \neq 0$.

On further expansion, Eq. (6) reads as

$$
i_{m}=a_{m 0} S_{0}+a_{m 1} S_{1}+a_{m 2} S_{2}+a_{m 3} S_{3},
$$

$$
m=0,1,2,3 \text {, }
$$




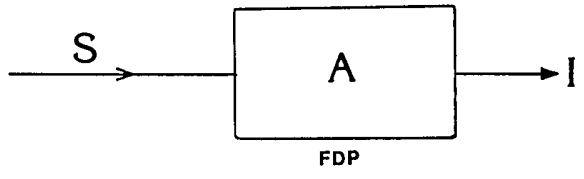

Fig. 1. FDP as a black box with the Stokes vector $\mathbf{S}$ of the incident light beam as input and the current vector $I$ as output. The instrument matrix $\mathbf{A}$ relates $\mathbf{I}$ to $\mathbf{S}$ by means of the linear relation $\mathbf{I}=\mathbf{A S}$.

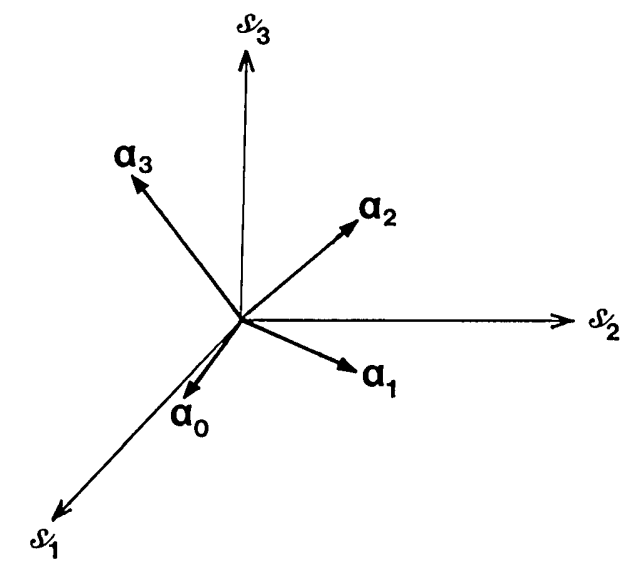

Fig. 2. A given FDP at a given wavelength is characterized by a set of four NPV's $\mathbf{a}_{0}, \mathbf{a}_{1}, \mathbf{a}_{2}$, and $\mathbf{a}_{3}$ (one for each of the four detectors) in the Stokes-Poincare $s_{1} s_{2} s_{3}$ space, where $s_{1}, s_{2}$, and $s_{3}$ are the normalized Stokes parameters.

where $a_{m n}(n=0,1,2,3)$ are the elements of $\mathbf{A}_{m}$. Next, Eq. (7) is normalized as follows:

$$
i_{m}=a_{m 0} S_{0}\left(1+\mathbf{a}_{m} \cdot \mathbf{s}\right),
$$

where

$$
\begin{aligned}
\mathbf{s} & =\left(S_{1} / S_{0}, S_{2} / S_{0}, S_{3} / S_{0}\right), \\
\mathbf{a}_{m} & =\left(a_{m 1} / a_{m 0}, a_{m 2} / a_{m 0}, a_{m 3} / a_{m 0}\right) .
\end{aligned}
$$

s of Eq. (9) is the pure polarization-state vector in the threedimensional Stokes-Poincaré space. ( $|\mathbf{s}|=1$ is the Poincaré sphere, which is the locus of all possible states of total polarization, and $|\mathbf{s}|<1$ corresponds to partially polarized light.) In this space (Fig. 2), the FDP is represented by the four normalized projection vectors (NPV's) $\mathbf{a}_{0}, \mathbf{a}_{1}, \mathbf{a}_{2}$, and $\mathbf{a}_{3}$. From the requirement that $\operatorname{det} \mathbf{A} \neq 0$, it follows that the endpoints of these four vectors must not lie in one plane (i.e., must form a pyramid of nonzero volume). In addition to $\mathbf{a}_{m}$ $(m=0,1,2,3)$, the FDP is also characterized by its response to incident unpolarized light. This is obtained by setting $\mathbf{s}$ $=0$ in Eq. (8):

$$
i_{m}=a_{m 0} S_{0}, \quad m=0,1,2,3 .
$$

Consequently, $a_{m 0}$ has the following meaning:

$$
a_{m 0}=i_{m} / S_{0}, \quad m=0,1,2,3,
$$

which is the (normalized) response of the $m$ th detector per unit power of incident unpolarized radiation.

Equation (8) determines the modulation of the output signal of the $m$ th detector as the input polarization vector $\mathbf{s}$ is allowed to scan the entire s space (inside and on the surface of the Poincare sphere), assuming a given constant $S_{0}$. It is apparent that the signal $i_{m}$ is maximum,

$$
i_{m \max }=a_{m 0} S_{0}\left(1+\left|\mathbf{a}_{m}\right|\right),
$$

when $\mathbf{s}$ is the unit vector in the direction of the projection vector $\mathbf{a}_{m}$. Likewise, $i_{m}$ is minimum,

$$
i_{m \min }=a_{m 0} S_{0}\left(1-\left|\mathbf{a}_{m}\right|\right),
$$

when $\mathbf{s}$ is the unit vector antiparallel to $\mathbf{a}_{m}$. The average signal level is given by Eq. (11) and is attained when $\mathbf{s}=\mathbf{0}$ (unpolarized light) or when $\mathbf{a}_{m} \cdot \mathbf{s}=0$, which places $\mathbf{s}$ in a plane through the origin orthogonal to $\mathbf{a}_{m}$ ending on or inside a great circle $\mathrm{C}$ (Fig. 3 ). More generally, a given signal level $i_{m}$ is attained when $\mathbf{a}_{m} \cdot \mathbf{s}=$ constant, which places the

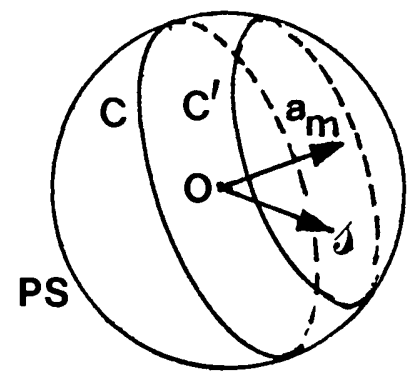

Fig. 3. The output signal of the $m$ th detector of the FDP is determined by the scalar product $\mathbf{a}_{m} \cdot \mathbf{s}$ of the input polarization vector $\mathbf{s}$ $=\left(s_{1}, s_{2}, s_{3}\right)$ and the NPV $\mathbf{a}_{m}$ of that detector in the Stokes-Poincaré space, according to Eq. (8). The average signal level is generated when the incident light is unpolarized, $\mathbf{s}=\mathbf{0}$ or, more generally, when $\mathbf{a}_{m} \cdot \mathbf{s}=0$, which places the endpoint of $\mathbf{s}$ in a plane orthogonal to $\mathrm{a}_{m}$ on or inside the great circle $\mathrm{C}$ of intersection of the plane with the Poincare sphere (PS: $|\mathbf{s}|=1$ ). The locus of all states producing the same signal level in the $m$ th detector, $\mathbf{a}_{m} \cdot \mathbf{s}=$ constant, is the circular disk defined by a circle $\mathrm{C}^{\prime}$ on the PS whose plane is normal to $\mathbf{a}_{m}$.

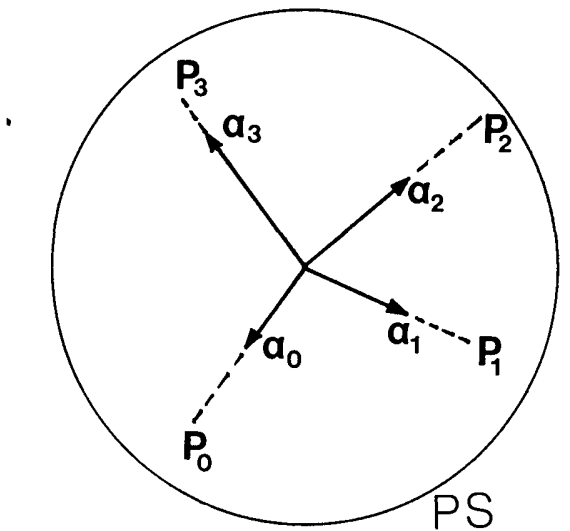

Fig. 4. A given FDP at a given wavelength is characterized by a set of four specific total polarization states $P_{0}, P_{1}, P_{2}$, and $P_{3}$ on the surface of the Poincare sphere PS. Each point $P_{m}$ is the point of intersection of the straight-line extension of the associated NPV $\mathbf{a}_{m}$ with the PS and represents the polarization state that produces the maximum possible output signal of the $m$ th detector. For each point $P_{m}$ there is a diametrically opposite point on the sphere $Q_{m}$, not shown, that represents the state that generates the minimum possible output signal of the $m$ th detector. 
endpoint of $\mathbf{s}$ in a plane normal to $\mathbf{a}_{m}$ that cuts the Poincaré sphere in a minor circle $\mathrm{C}^{\prime}$ (Fig. 3 ).

If $S_{0}$ is known, only three projections (signals) are sufficient to fix s completely by the point of intersection of three different planes, each of which is orthogonal to the corresponding NPV, $\mathbf{a}_{m}$. A unique point of intersection is guaranteed if the three NPV's are not coplanar.

The points of intersection of the extensions of the four NPV's $\mathbf{a}_{m}(m=0,1,2,3)$ with the Poincaré sphere define four total-polarization states $P_{m}$ (Fig. 4) that provide an interesting characterization of the FDP. From the preceding discussion, $P_{m}$ is the polarization state of incident light that produces the maximum possible output signal level $i_{m}$ of the $m$ th detector. Four associated points $Q_{m}$, each of which is diagonally opposite a corresponding point $P_{m}$, give an equivalent representation on the Poincaré sphere, in terms of the minimum signals.

\section{EIGHT CONSTRAINTS ON THE ELEMENTS OF THE FOUR-DETECTOR PHOTOPOLARIMETER INSTRUMENT MATRIX A}

Because each detector is absorptive, $i_{m}$ is always nonnegative:

$$
i_{m} \geq 0 \text {. }
$$

(A negative current is meaningless in the present context of the FDP.) And, because $S_{0}>0$, it follows from Eq. (11) that

$$
a_{m 0}>0, \quad m=0,1,2,3 .
$$

Inequality (16) indicates that each and every element in the first column of $\mathbf{A}$ must always be positive.

From relations (14)-(16) one concludes that

$$
\left|\mathbf{a}_{m}\right| \leq 1, \quad m=0,1,2,3,
$$

which indicates that the length of each NPV is generally $<1$ and may reach 1 as a limiting case. ${ }^{7}$ This second set of four inequalities can be put into the expanded form

$$
\left(a_{m 1}^{2}+a_{m 2}^{2}+a_{m 3}^{2}\right)^{1 / 2} \leq a_{m 0}, \quad m=0,1,2,3
$$

if Eq. (10) is used. The eight inequalities (16) and (18) are satisfied by the elements $a_{m n}$ of the instrument matrix of any FDP.

\section{TWO ZERO ELEMENTS OF THE INSTRUMENT MATRIX A OF A FOUR- DETECTOR PHOTOPOLARIMETER USING ISOTROPIC DETECTORS}

For a FDP using optically isotropic photodetectors (for which the $p$ and $s$ linear polarizations, parallel and perpendicular to the plane of incidence, are the eigenpolarizations of reflection), the instrument matrix $\mathbf{A}$ is given by the product $^{3}$

$\mathbf{A}=\left[\begin{array}{llll}k_{0} & 0 & 0 & 0 \\ 0 & k_{1} & 0 & 0 \\ 0 & 0 & k_{2} & 0 \\ 0 & 0 & 0 & k_{3}\end{array}\right]\left[\begin{array}{rrrr}1 & -1 & 0 & 0 \\ 0 & 1 & -1 & 0 \\ 0 & 0 & 1 & -1 \\ 0 & 0 & 0 & 1\end{array}\right]\left[\begin{array}{llll}1 & 0 & 0 & 0 \\ f_{10} & f_{11} & 0 & 0 \\ f_{20} & f_{21} & f_{22} & f_{23} \\ f_{30} & f_{31} & f_{32} & f_{33}\end{array}\right]$, where the first matrix is the responsivity matrix $\mathbf{K}$ and the elements $f_{i j}$ of the third matrix $\mathbf{F}$ are given by Eqs. (11) of Ref. 3. Carrying out the matrix multiplications of Eq. (19), we find that

$$
\begin{aligned}
& a_{02}=0, \\
& a_{03}=0,
\end{aligned}
$$

i.e., the last two elements of the first row of $\mathbf{A}$ are zero.

It should be noted that Eq. (21) always holds, irrespective of the reference axes at the input of the FDP with respect to which $\mathbf{S}$ and $\mathbf{A}$ are measured. However, Eq. (20) holds only when those reference axes are aligned with the directions parallel $\left(p_{0}\right)$ and perpendicular $\left(s_{0}\right)$ to the plane of incidence for light reflection at the first detector $\left(D_{0}\right)$.

\section{TEST OF THE CONSTRAINTS ON THE ELEMENTS OF A FOR A SPECIFIC MEASURED MATRIX}

For a FDP using four Si P-I-N photodetectors and operating at $\lambda=632.8 \mathrm{~nm}$, the following matrix:

$$
\mathbf{A}=\left[\begin{array}{rrrr}
0.746 & 0.157 & -0.071 & -0.001 \\
2.433 & -1.180 & 0.516 & 0.447 \\
0.694 & -0.244 & 0.137 & -0.405 \\
2.597 & -1.934 & -0.296 & -0.429
\end{array}\right],
$$

was recently given, ${ }^{6}$ free of imperfections in the calibration polarization optics. This matrix satisfies the following conditions:

(1) All elements of its first column are positive, in accord with inequality (16).

(2) The lengths of the four NPV's, $\left|\mathbf{a}_{0}\right|=0.231,\left|\mathbf{a}_{1}\right|=$ $0.560,\left|\mathbf{a}_{2}\right|=0.709$, and $\left|\mathbf{a}_{3}\right|=0.771$, are all $<1$, in accord with inequality (17).

(3) $a_{03}=-0.001 \approx 0$, in good agreement with Eq. (21).

(4) Because A of Eq. (22) was measured with respect to an external laboratory coordinate frame (and not the directions $p_{0}$ and $s_{0}$ parallel and normal to the first plane of incidence), $a_{02}=-0.071 \neq 0$. However, $a_{02}$ can be forced $=$ 0 [hence Eq. (20) is satisfied] by an appropriate coordinate rotation, as shown in Ref. 6.

Finally, we note that the NPV's associated with our prototype FDP of four Si detectors and its A matrix of Eq. (22) satisfy the following additional set of three inequalities:

$$
\left|\mathbf{a}_{0}\right|<\left|\mathbf{a}_{1}\right|<\left|\mathbf{a}_{2}\right|<\left|\mathbf{a}_{3}\right| .
$$

Thus the length of the NPV increases monotonically from detector to detector as we move downstream in the FDP, which indicates the increasingly polarizing effect of successive reflections.

However, inequalities (23) do not apply in all cases. ${ }^{8}$ For example, one or more may be violated near the conditions of 
singularity of the $\mathbf{A}$ matrix $^{3}$ (e.g., when the rotation angle of the plane of incidence is, say, $80^{\circ}$ ).

\section{PHYSICAL MEANING OF THE COLUMNS OF} A

A can be written in terms of its columns as

$$
A=\left[\begin{array}{llll}
C_{0} & C_{1} & C_{2} & C_{3}
\end{array}\right]
$$

It is convenient to define the normalized response of the FDP for any input polarization state by

$$
\begin{aligned}
\boldsymbol{J} & =\mathbf{I} / S_{0} \\
& =\left[\begin{array}{llll}
\mathbf{C}_{0} & \mathbf{C}_{1} & \mathbf{C}_{2} & \mathbf{C}_{3}
\end{array}\right] \mathbf{S} / S_{0},
\end{aligned}
$$

which is the output current vector per unit power of the incident polarized radiation. It follows from Eq. (26), or from Eq. (12), that

$$
\mathbf{C}_{0}=\mathcal{J}_{\text {UPL }}
$$

i.e., the first column of the instrument matrix $\mathbf{A}$ specifies the normalized response of the FDP to incident unpolarized light (UPL).

Next let us consider the response of the FDP to the same orthogonal pairs of states that are employed in the phenomenological definitions of the Stokes parameters. ${ }^{4,5}$ These are

(1) The orthogonal linear polarizations at 0 and $90^{\circ}$ azimuth (L0 and L90),

(2) The orthogonal linear polarizations at $45^{\circ}$ and $135^{\circ}$ azimuths (L45, L135),

(3) The orthogonal right- and left-handed circular polarizations (RCP and LCP, respectively), as shown in Fig. 5.

For the first pair of states, the Stokes vectors are

$$
\begin{aligned}
\mathbf{S}_{\mathrm{L} 0} & =S_{0}\left[\begin{array}{lll}
1 & 1 & 0
\end{array}\right]^{t}, \\
\mathbf{S}_{\mathrm{L} 90} & =S_{0}\left[\begin{array}{llll}
1 & -1 & 0 & 0
\end{array}\right]^{t},
\end{aligned}
$$

and the associated FDP's normalized responses [from Eq. (26)] are

$$
\begin{aligned}
\mathcal{J}_{\mathrm{L} 0} & =\mathbf{C}_{0}+\mathbf{C}_{1}, \\
\mathcal{J}_{\mathrm{L} 90} & =\mathbf{C}_{0}-\mathbf{C}_{1} .
\end{aligned}
$$

From Eqs. (29), it immediately follows that

$$
\mathbf{C}_{1}=1 / 2\left(\mathcal{J}_{\mathrm{L} 0}-\mathcal{J}_{\mathrm{L} 90}\right),
$$

which shows that the second column of the instrument matrix A represents the FDP's differential normalized response to incident orthogonal linear polarizations at 0 and $90^{\circ}$ azimuths.

For the second pair of states, the Stokes vectors are

$$
\begin{aligned}
& \mathbf{S}_{\mathrm{L} 45}=S_{0}\left[\begin{array}{llll}
1 & 0 & 1 & 0
\end{array}\right]^{t}, \\
& \mathbf{S}_{\mathrm{L} 135}=S_{0}\left[\begin{array}{llll}
1 & 0 & -1 & 0
\end{array}\right]^{t},
\end{aligned}
$$

and the associated FDP's normalized responses [from Eq. (26)] are
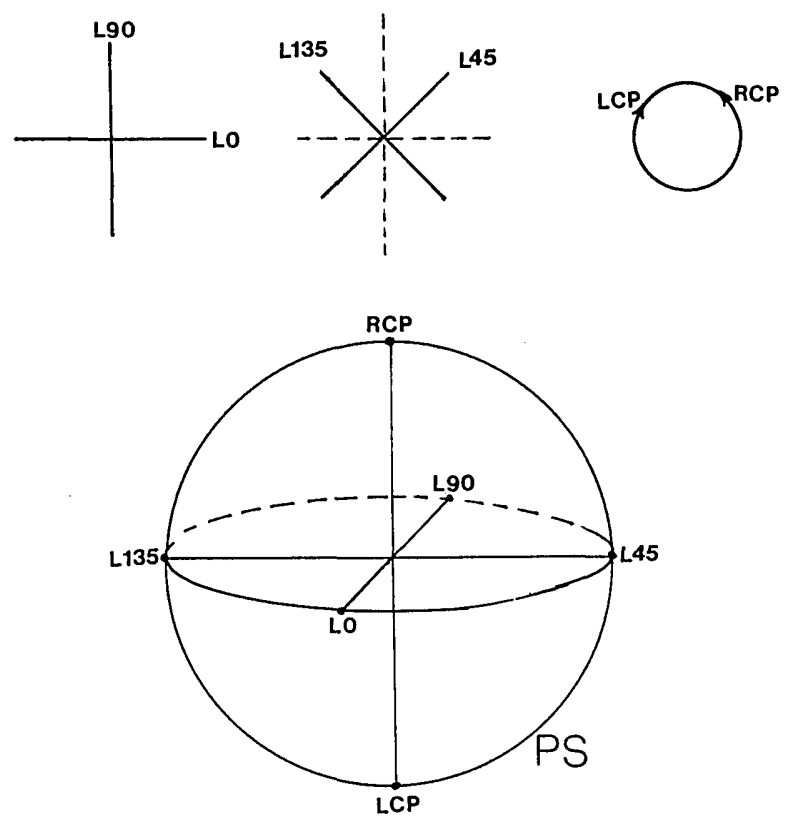

Fig. 5. The second, third, and fourth columns of the instrument matrix $\mathbf{A}$ of the FDP represent the differential normalized responses of the FDP for incident light polarized in the indicated pairs of orthogonal states: (1) L0 and L90, the linear polarizations at 0 and $90^{\circ}$ azimuths, (2) L45 and L135, the linear polarizations at $45^{\circ}$ and $135^{\circ}$ azimuths, and (3) RCP and LCP, the right- and left-handed circular polarization states, respectively. The first column of A represents the average response to any pair of orthogonal states.

$$
\begin{gathered}
\mathcal{J}_{\mathrm{L} 45}=\mathbf{C}_{0}+\mathbf{C}_{2}, \\
\mathcal{J}_{\mathrm{L} 135}=\mathbf{C}_{0}-\mathbf{C}_{2} .
\end{gathered}
$$

From Eqs. (32) it follows that

$$
\mathbf{C}_{2}=1 / 2\left(\mathcal{J}_{\mathrm{L} 45}-\mathcal{J}_{\mathrm{L} 135}\right),
$$

which indicates that the third column of the instrument matrix A represents the FDP's differential normalized response to incident orthogonal linear polarizations at $45^{\circ}$ and $135^{\circ}$ azimuths.

Finally, for the third pair of states, the Stokes vectors are

$$
\begin{aligned}
& \mathbf{S}_{\mathrm{RCP}}=S_{0}\left[\begin{array}{llll}
1 & 0 & 0 & 1
\end{array}\right]^{t}, \\
& \mathbf{S}_{\mathrm{LCP}}=S_{0}\left[\begin{array}{llll}
1 & 0 & 0 & -1
\end{array}\right]^{t},
\end{aligned}
$$

and the associated FDP's normalized responses [from Eq. (26)] are

$$
\begin{aligned}
& \mathcal{J}_{\mathrm{RCP}}=\mathbf{C}_{0}+\mathbf{C}_{3}, \\
& \mathcal{J}_{\mathrm{LCP}}=\mathbf{C}_{0}-\mathbf{C}_{3} .
\end{aligned}
$$

From Eqs. (35) we obtain

$$
\mathbf{C}_{3}=1 / 2\left(\mathcal{J}_{\mathrm{RCP}}-\mathcal{J}_{\mathrm{LCP}}\right),
$$

which shows that the fourth (last) column of the instrument matrix A represents the FDP's differential response to the incident right- and left-handed circular polarization states.

Before concluding this section, we note that Eqs. (29), (32), and (35) also give 


$$
\begin{aligned}
\mathbf{C}_{0} & =1 / 2\left(\mathcal{J}_{\mathrm{L} 0}+\mathcal{J}_{\mathrm{L} 90}\right)=1 / 2\left(\mathcal{J}_{\mathrm{L} 45}+\mathcal{I}_{\mathrm{L} 135}\right) \\
& =1 / 2\left(\mathcal{J}_{\mathrm{RCP}}+\mathcal{J}_{\mathrm{LCP}}\right) .
\end{aligned}
$$

Equations (37) lead to the following general result:

$$
\mathbf{C}_{0}=1 / 2\left(\mathcal{J}_{\mathrm{S}}+\mathcal{J}_{\mathrm{OS}}\right),
$$

which states that the first column of $\mathbf{A}$ also represents the average of the normalized responses of the FDP to any pair of orthogonal polarization states (denoted S and OS).

\section{SUMMARY}

In this paper we have provided new insight into the meaning of the rows and columns of the instrument matrix $\mathbf{A}$ of the FDP, which matrix links the output current vector $I$ and the input Stokes vector $\mathbf{S}$ according to $\mathbf{I}=\mathbf{A S}$. In particular, the rows of $\mathbf{A}$ define four projection operators and an associated set of four NPV's $\mathbf{a}_{m}$ with certain geometrical interpretation in the three-dimensional Stokes-Poincaré space. In general, each NPV is of length $\leq 1$, and the equality may hold only as a limiting case.

The first column of A represents the FDP's normalized response to incident unpolarized light, and each and every one of its elements is $>0$. It also represents the average normalized response of the FDP to incident light, which is polarized in any pair of orthogonal states.

The second, third, and fourth column of A represent the FDP's differential normalized responses to (1) orthogonal linear polarizations at 0 and $90^{\circ}$ azimuths, (2) orthogonal linear polarizations at $45^{\circ}$ and $135^{\circ}$ azimuths, and (3) the orthogonal right- and left-handed circular polarization states, respectively. These are the same three sets of orthogonal pairs of states that are used in the phenomenological definitions of the Stokes parameters.

\section{ACKNOWLEDGMENTS}

This research is supported by the National Science Foundation under grant ECS8520035 and was presented at the 1988 Annual Meeting of the Optical Society of America in Santa Clara, California, October 30-November 4, 1988.

\section{REFERENCES AND NOTES}

1. R. M. A. Azzam, "Arrangement of four photodetectors for measuring the state of polarization of light," Opt. Lett. 10, 309-311 (1985); U.S. Patent 4,681,450 (July 21, 1987).

2. R. M. A. Azzam, E. Masetti, I. M. Elminyawi, and F. G. Grosz, "Construction, calibration, and testing of a four-detector photopolarimeter," Rev. Sci. Instrum. 59, 84-88 (1988).

3. R. M. A. Azzam, I. M. Elminyawi, and A. M. El-Saba, "General analysis and optimization of the four-detector photopolarimeter," J. Opt. Soc. Am. A 5, 681-689 (1988).

4. M. Born and E. Wolf, Principles of Optics (Pergamon, New York, 1975), p. 554.

5. R. M. A. Azzam and N. M. Bashara, Ellipsometry and Polarized Light (North-Holland, Amsterdam, 1987), p. 55.

6. R. M. A. Azzam and A. G. Lopez, "Accurate calibration of the four-detector photopolarimeter with imperfect polarizing optical elements," J. Opt. Soc. Am. A 6, 1513-1521 (1989).

7. In a conventional photopolarimeter an ideal linear polarizer (analyzer) is almost always placed as the last element of the optical train, adjacent to the photodetector. Equations similar to Eqs. (7) and (8) hold [see, e.g., R. M. A. Azzam, "Measurement of the Stokes parameters of light: a unified analysis of Fourier photopolarimetry," Optik 52, 253-256 (1979)]. However, in this case, the NPV $\mathbf{a}_{m}$ is invariably of unit length, $\left|\mathbf{a}_{m}\right|=1$. Inequality (17) represents another important distinction that sets the FDP apart from conventional instruments.

8. If optimum rotations of the plane of incidence, and reflection at equal $\left(65^{\circ}\right)$ angles from three identical detectors, are assumed, we find, using the analysis of Ref. 3 , that inequalities (23) hold at all wavelengths $(200-800 \mathrm{~nm}$ ) for Si detectors coated with 200 $\mathrm{nm} \mathrm{SiO}_{2}$ film. Surprisingly, for a thin $20-\mathrm{nm}$ film, the inequalities are violated at shorter wavelengths $(<400 \mathrm{~nm})$. This runs somewhat counter to intuition. 